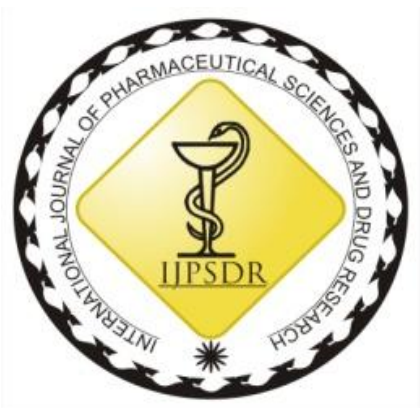

\author{
RESEARCH ARTICLE
}

ISSN: 0975-248X

CODEN (USA): IJPSPP

(c) EY-NG-SA

\title{
Comparative Antidiabetic Potential Assessment of Herbal Formulation of Gymnemic Acid and Curcumin
}

\author{
Alok Shukla1, Mridula Sachan², Papiya Bigoniya ${ }^{3 *}$ \\ ${ }^{1}$ Amity Institute of Pharmacy, Amity University, Near Malhaur Railway Station, Gomti Nagar Extn, Lucknow, \\ Uttar Pradesh, India \\ ${ }^{2}$ Radharaman College of Pharmacy, Radharaman Group of Institutes, Ratibad, Bhopal, Madhya Pradesh, India \\ ${ }^{3}$ DSKM College of Pharmacy, RKDF University, Gandhi Nagar, Bhopal, Madhya Pradesh, India
}

Copyright (C) 2019 Alok Shukla et al. This is an open access article distributed under the terms of the Creative Commons AttributionNonCommercial-ShareAlike 4.0 International License which allows others to remix, tweak, and build upon the work non-commercially, as long as the author is credited and the new creations are licensed under the identical terms.

\begin{abstract}
Standardization of polyherbal formulations with respect to bioactive phytocompounds is the need of the time for registration and marketing authorization in developed countries. This has prompted to prepare and evaluate a standardized bioactive phyotcompounds containing formulation. The study aims at development and screening of a standardized antidiabetic suspension containing active isolated phytoconstituents targeting better therapeutic effect with reduced bioburden. Suspension of isolated gymnemic acid and curcumin (GCS) was prepared, evaluated and authenticated by TLC and HPTLC. Antidiabetic efficacy of GCS was screened against alloxan induced diabetes on rats following 28 days of treatment comparative to Hyponidd tablet and Madhumehari granules. Body weight, relative organ weight, blood glucose, cholesterol (TC), triglyceride (TG), high density lipoprotein (HDL), low density lipoprotein (LDL) and very low density lipoprotein (VLDL) level was measured. The formulation having pH 6.0, refractive index 1.41 and $45.58 \mathrm{mg} / \mathrm{ml}$ total solid content showed high alcohol and water soluble extractive value. The GCS treatment normalized liver and kidney weight, decreased body weight gain, TC, TG, LDL and VLDL level along with an increase in HDL level. Study outcome signifies similar antidiabetic potential of standardized formulation GCS compared to marketed Polyherbal formulation with antihyperlipidemic activity signifying as a promising natural and safe remedy for the prevention of diabetic complications.
\end{abstract}

Keywords: Gymnemic acid, Curcumin, Polyherbal formulation, antidiabetic, antihyperlipidemic.

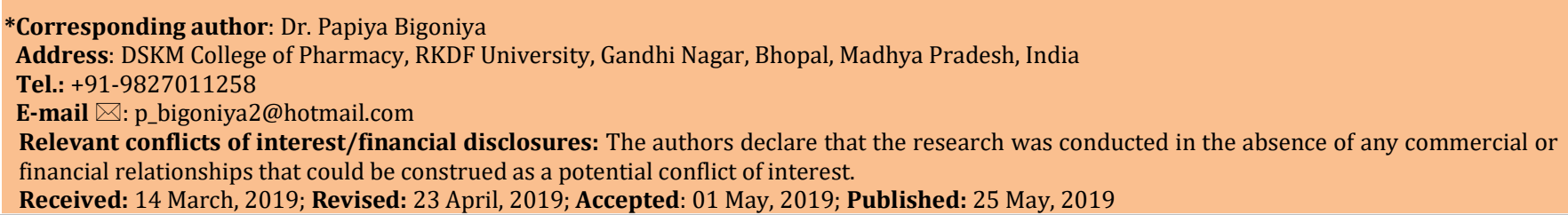

\section{INTRODUCTION}

Well established scientific database on active substances characterization along with establishment of safety, efficacy, and quality of traditional medicinal products are required for marketing authorization in most of the developed countries. Nowadays, though herbal medicine preparations are manufactured and sold extensively in India but are allowed exporting in developed countries only as food supplements. The traditional medicines are not sufficiently qualified to 
reach the market of developed countries as drug product due to inadequate studies on active ingredient identification and quality control. [1] India though has one of the oldest systems of traditional medicine but with just $2 \%$ share in the global herbal export market are not able to exploit the opportunities of the emerging demand. Development of traditional medicine formulation enabled characterization and quantification of bioactive or marker phytocompounds can provide global regulatory acceptance. [2] Considering this present scenario, focus on the development of standardized herbal formulation with well established bioactive phytocompounds is need of the time.

With the rise in socio-economic and living standards, diabetes has become the country's fastest growing disease burden. India currently represents $49 \%$ of the world's diabetes patients, with an estimated 72 million cases in 2017, which is expected to double by 2025. [3] Management of diabetes without long term side effect is still a challenge as currently available synthetic antidiabetic drugs can produce serious side effects such as hypoglycemic coma and hepatorenal disturbances. [4] The herbal antidiabetic products are extensively formulated commercially because of easy availability, affordability and fewer side effects as compared to synthetic antidiabetic drugs. [5] Compared to the single herb, the polyherbal formulation has better and extended therapeutic potential. The minute quantity of active phytochemical constituents present in the formulation are insufficient to achieve the desired therapeutic effects, but when combined surely give better therapeutic effect but is also associated with higher bioburden and increased chances of adverse effect. [6] Isolated phytocompounds, when used in rationalized dose, can produce similar therapeutic effect while enduring less metabolic burden due to relative easy elimination. Standardization and authentication of phytocompounds are the essential aspects of quality and safety consistency of the polyherbal formulation as this can minimize batch to batch variation with safety, efficacy, and quality assurance enhancing acceptability. [7]

This study was aimed at preparation and standardization of an herbal formulation containing gymnemic acid and curcumin isolated from Gymnema sylvestre (Gurmar) and Curcuma longa (Haldi) known for potential antidiabetic activity. Two plants having sound ethnopharmacological and scientific citation background for antidiabetic activity was thus selected along with the most bioactive compound. The study encompasses enrichment and authentication of phytoactive compounds gymnemic acid and curcumin, development of a standardized formulation and in vivo screening for antidiabetic activity on rats compared with the marketed polyherbal formulations containing both the selected plants along with other herbs.
Survey on the Composition of the Marketed Antidiabetic Herbal Formulation

A market survey was conducted on Ayurvedic formulations available in different parts of Bhopal city, i.e., New Market, Jawahar Chowk, 10 number market, and Hamidia road. Based on the sale value of the formulations, comparative assessment was done considering the formulation brand name, manufacturer, composition, market reputation, and sale volume.

Selection of two Potential Formulations for the Proposed Study

Details of total 25 products were compiled having good sale value, out of these 10 were tablet, 2 capsules, 4 rasas, 3 granules, and 6 powder products containing 2 to 15 plants. The most commonly used plants were found to be Gymnema sylvestre (Gurmar), Momordica charantia (Karela), Emblica officinalis (Amla), Curcuma longa (Haldi), Syzygium cumini (Jamun) and Tinospora cordifolia (Giloy). The formulations selected for comparative study were Hyponid tablet containing 11 plants and Madhumehari granules having 14 plants along with shilajit that had higher sale value and market reputation. Common ingredients in both formulations were haldi, amla, gurmar, kalela, and gelogy. Gymnema sylvestre (Family Asclepiadaceae) and Curcuma longa (Family Zingiberaceae) was selected for isolation and enrichment of gymnemic acid and curcumin with reported antidiabetic property. ${ }^{[8-9]}$

Chemicals

Aventis Pharma Ltd provided glibenclamide (Daonil) as a gift sample. Standard Gymnemic acid and curcumin were purchased from Natural Remedy Pvt. Ltd., Bangalore.

\section{Collection and Authentication of Plant Material}

The plant materials G. sylvester leaves and C. longa rhizome were collected in the month of Nov-Dec 2010 from Vindhya herbals Bhopal, M. P. Plant materials were authenticated by Dr. Ziaul Hasan Assistant Professor, Department of Botany, Safia Science College, Bhopal, and a specimen voucher no.189,190/Bot/Safia/10 for future reference. The leaves of G. sylvester and rhizomes of C. longa were dried in the shade for 15 days, ground to coarse powder and kept in airtight container.

Extraction of Plant Material

G. sylvestre leaf powder and turmeric powder was subjected to continuous soxhlet extraction with $80 \%$ ethanol separately. The filtrate was evaporated using a rotatory evaporator, dried and yield was calculated. [10] Isolation of Gymnemic Acid from G. sylvestre Ethanolic Extract

G. sylvestre ethanolic extract was dissolved in 50\% (v/v) ethanol, $\mathrm{KOH}$ was added and heated on a boiling water bath under reflux for an hour and cooled. To this $12 \mathrm{~N}$, hydrochloric acid was added and heated on a water bath. After cooling the $\mathrm{pH}$ was adjusted to 7.5-8.5 with $11 \% \mathrm{KOH}$. This solution was dissolved with $50 \%$ (v/v) 
ethanol, filtered and evaporated to obtain crude gymnemic acid fraction. [11]

Purification of Gymnemic Acid by Preparative Thin Layer Chromatography (TLC)

Silica gel $\mathrm{G}_{254}$ coated plates were activated at $110^{\circ} \mathrm{C}$ before use. Crude gymnemic acid fraction dissolved in methanol was spotted, and the plates were placed into the developing chamber containing solvent system chloroform: methanol: ethyl acetate 5:1:1. The spot corresponding to retention factor $\left(R_{f}\right)$ of gymnemic were scraped from 12 plates, combined, dissolved in methanol, filtered, concentrated, and lyophilized to obtain purified gymnemic acid. [10]

Isolation of Curcumin from C. longa Ethanolic Extract Ethanolic extract of curcumin was dissolved in benzene and extracted with an equal volume of $0.1 \% \mathrm{NaOH}$. The combined alkaline extract was acidified with dilute $\mathrm{HCl}$ acid that followed with the formation of yellow colored precipitate which was concentrated on a water bath. The resinous material agglomerated and form a lumpy mass that was filtered, concentrated, and lyophilized to get curcumin. [12]

\section{Anti-diabetic Formulation Development and} Evaluation

Accurately weighted lyophilized isolated gymnamic acid and curcumin (to contain $30 \mathrm{mg} / \mathrm{ml}$ each in the final formulation) were mixed with minimum quantity $1 \%$ of tween 80 in distilled water continuously to get a homogenous suspension of Gymnamic acid and curcumin (GCS) and mixed with glycerin containing xanthan gum. The final volume of GCS was made up with sorbitol $(70 \%)$, preserved in a sealed amber colored bottle. Hyponid and Madhumehari samples, and the GCS were evaluated for $\mathrm{pH}$, solid content, refractive index, total ash, water soluble ash, acid insoluble ash, alcohol soluble extractive, and water soluble extractive values as per the method described in Indian Pharmacopoeia and WHO guidelines on quality control methods for medicinal plant materials. [13]

\section{Authentication of Gymnemic Acid and Curcumin TLC}

Isolated and standard gymnemic acid was dissolved in methanol, filtered and spotted in activated silica gel $\mathrm{G}_{254}$ coated plates. CGS, Hyponid and Madhumehari samples were extracted with methanol, filtered, concentrated and spotted. Solvent system chloroform: methanol: ethyl acetate in 5:1:1 ratio was used. Isolated and standard curcumin along with CGS, Hyponid, and Madhumehari samples were extracted with ethanol and chloroform: ethanol: acetic acid in 94:5:1 ratio was applied as the solvent system. [14]

\section{High Performance Thin Layer Chromatography} (HPTLC)

HPTLC was performed on a CAMAG system which includes a pre-coated plate (silica gel $60 \mathrm{~F}_{254}$, Merck), high pressure sample injector of $100 \mu$ capacity, an HPTLC development chamber and scanning UVcabinet. Spotting was done by the auto-injector on a
$10 \times 10 \mathrm{~cm}$ pre-coated silica plate with $2 \mu \mathrm{l}$ of the sample for banding. Developed plates were dried and observed in the UV-cabinet at $254 \mathrm{~nm}$ wavelength. Data analysis was done with the help of the CAMAG- Linomat 5 software system. Solvent system applied was same as used for TLC. [14] Parallel plates were run for standard gymnemic acid, standard curcumin, isolated gymnemic acid, isolated curcumin, CGS, Hyponidd and Madhumehari samples.

Antidiabetic Study

Experimental Animals

Laboratory breed adult Wistar rats (150-200 g) of either sex maintained under standard laboratory conditions at $22 \pm 2{ }^{\circ} \mathrm{C}$; relative humidity $65 \pm 5 \%$ and photoperiod (12 hr dark and light) were used. The animals were housed in paddy husk bedded polycarbonate cages and fed with standard pallet balanced diet and purified water ad libitum. The study was performed with due permission from the Institutional Animal Ethical Committee (Approval No. IAEC/RCP-06/2011), and all care was provided as per the guideline of CPCSEA, New Delhi.

Dose Preparation

GCS was administered orally at the dose of $0.5 \mathrm{ml} / 100$ gm $(75 \mathrm{mg} / \mathrm{kg}$ each of gymnemic acid and curcumin in $15 \mathrm{mg} / 0.5 \mathrm{ml}$ of GCS). Hyponidd tablet and Madhumehari granules were separately triturated with a minimum quantity of $1 \%$ Tween 80 to get homogenous suspension and volume make up with distilled water. Both the formulations were administered orally at a dose of $200 \mathrm{mg} / \mathrm{kg}$ in a final volume of $0.5 \mathrm{ml} / 100 \mathrm{gm}$. Glibenclamide suspended in $1 \%$ Tween 80 in distilled water was given at $5 \mathrm{mg} / \mathrm{kg}$ body weight daily by gastric intubation. [15]

\section{Induction of Diabetes}

Alloxan monohydrate dissolved in sterile normal saline was injected intraperitoneally at $150 \mathrm{mg} / \mathrm{kg}$ dose to overnight fasted rats. Blood samples were collected before the administration of alloxan and after 5 days. Rats with fasting blood sugar level more than 150 $\mathrm{mg} / \mathrm{dL}$ were considered to be diabetic and were selected for study. [16]

\section{Study Protocol}

Animals were randomly divided into 5 groups of six rats each. Group, I diabetic control was administered with $1 \%$ tween 80 solutions $(0.5 \mathrm{ml} / 100 \mathrm{gm}, p .0)$, Group II was administered with glibenclamide (5 mg/kg), Group III GCS (150 mg/kg), Group IV Hyponidd (250 $\mathrm{mg} / \mathrm{kg}$ ) and Group V Madhumehari $(250 \mathrm{mg} / \mathrm{kg}$ ) once in a day for 28 days. Body weight of all the animals was recorded at $0,10^{\text {th }}, 20^{\text {th }}$ and $28^{\text {th }}$ day and percent change in body weight was calculated. Blood glucose level was recorded at $0,10^{\text {th }}, 20^{\text {th }}$ and $28^{\text {th }}$ day after overnight fasting. After the completion of treatment period animals were sacrificed and blood samples collected by cardiac puncture, kept at room temperature for $30 \mathrm{~min}$ and centrifuged at $3000 \mathrm{rpm}$ for $20 \mathrm{~min}$. Serum was analyzed for various biochemical parameters such as total cholesterol (TC), triglyceride (TG), high density 
lipoprotein (HDL) and low density lipoprotein (LDL) by using Biosystem A-25 fully automatic biochemistry analyzer (Span Diagnostics Ltd., India) and serum VLDL level was calculated by using formula as described by Friedewald. [17] Liver and kidneys were isolated, kept in ice cold saline and weighted after blotting in filter paper. Relative organ weight (weight of organ/100 gm of body weight) was calculated and recorded.

\section{Statistical Analysis}

Experimental data were analyzed using one way ANOVA followed by Turkey-Kramer multiple comparison tests. The p-value less than 0.05 were considered statistically significant. Graph Pad Prism Version 3.02 was used for statistical calculations.

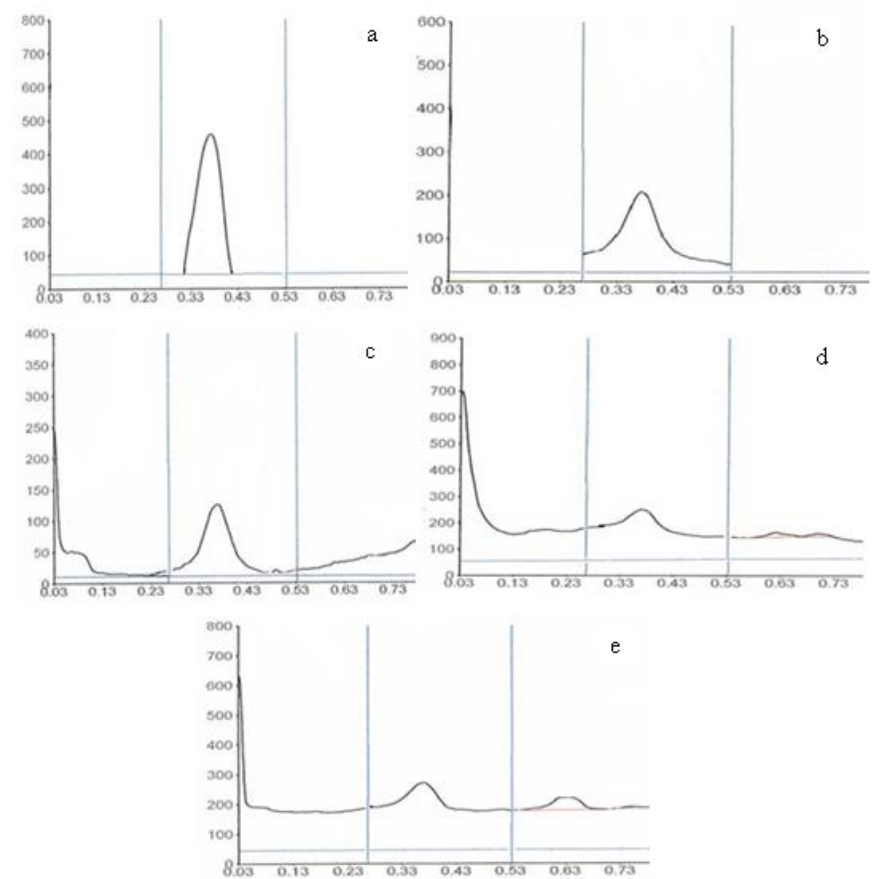

Fig. 1: HPTLC of gymnemic acid a: standard $(R t=0.37)$, b: isolated $(\mathrm{Rt}==0.38)$, c: GCS $(\mathrm{Rt}=0.38)$, d: Hyponidd $(\mathrm{Rt}=0.38)$ and $\mathrm{e}$ : madhumehari $(\mathrm{Rt}=\mathbf{0 . 3 9})$

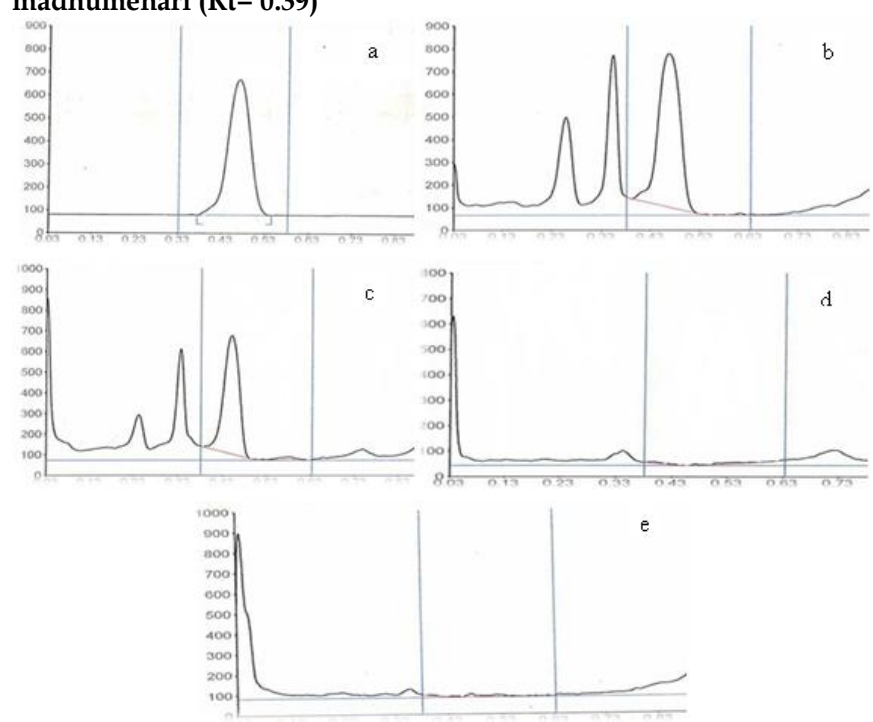

Fig. 2: HPTLC of curcumin a: standard (Rt $=0.47)$, b: isolated $(\mathrm{Rt}=$ 0.46), c: GCS (Rt $=0.46)$, d: Hyponidd (no visible peak) and e: Madhumehari (no visible peak)

\section{RESULTS}

Anti-diabetic Formulation Development and Evaluation

The gymnemic acid and curcumin suspension were of yellowish brown color with characteristic odor and sweet taste. The formulation showed $\mathrm{pH} 6.0$, refractive index 1.41 and total solid content as $45.58 \mathrm{mg} / \mathrm{ml}$. The value of total ash, acid insoluble and water soluble ash, and alcohol and water soluble extractive value for GCS, Hyponid, and Madhumehari is presented in Table 1. Total ash, acid insoluble and water soluble ash value was high in Hyponid tablet compared to Madhumehari granules. The GCS showed high alcohol and water soluble extractive value compared to Hyponid and Madhumehari both.

\section{Characterization of Gymnemic Acid and Curcumin}

In co-TLC, isolated gymnemic acid, GCS, Hyponid, and Madhumehari showed $\mathrm{R}_{\mathrm{f}}$ value $0.38,0.38,0.38$ and 0.39 respective to 0.37 of standard gymnemic acid. Standard curcumin showed $R_{t}$ at 0.47 whereas isolated curcumin, GCS, Hyponid and Madhumehari showed $R_{\mathrm{f}}$ at 0.46 , $0.46,0.47,0.46$ respectively validating the presence of the gymnemic acid and curcumin in all the three formulations (Table 2).

The HPTLC retention time $\left(\mathrm{R}_{\mathrm{t}}\right)$ of the standard gymnemic acid is reported to be 0.37 . The isolated gymnemic acid sample, GCS, Hyponid, and Madhumehari showed $R_{t}$ of $0.38,0.38,0.38$ and 0.39 respectively confirming presence of gymnemic acid. The $R_{t}$ of the standard curcumin was found to be 0.47 , whereas isolated curcumin and GCS showed $R_{t}$ of 0.46 and 0.46 confirming the presence. The test samples of both the marketed formulation (Hyponid and Madhumehari) showed absence of a peak at the corresponding $\mathrm{R}_{\mathrm{t}}$ of standard curcumin (Fig. 1 and 2).

\section{Antidiabetic Effect}

\section{Effect on Body Weight and Blood Glucose Level}

The body weight of vehicle treated rat showed average $12.43 \%$ weight gain whereas the rats of the diabetic control group showed gradual (-9.7\%) decrease during the 28 days treatment period. Glibenclamide, GCS, Hyponid, and Madhumehari showed respectively 7.32, $3.15,1.50$ and $3.14 \%$ increase in body weight. Modest hyperglycemia was developed in the diabetic control group with $13.92 \%$ increase in blood glucose after the $28^{\text {th }}$ day whereas glibenclamide, GCS, Hyponid and Madhumehari effectively suppressed blood glucose by $42.85,19.50,16.50$ and $17.53 \%$ compared to zero day value (Fig. 3).

\section{Effect on Relative Liver and Kidney Weight}

The diabetic control group showed significantly $(\mathrm{p}<0.05)$ decreased the relative weight of liver and increased kidney relative weight. GCS, Hyponid, and Madhumehari showed normalization of the relative weight of the liver and kidney as they showed nonsignificant changes compared to the vehicle control group (Table 3). 
Alok Shukla et al. / Antidiabetic Potential Assessment of Herbal Formulation of Gymnemic Acid.

Table 1: Physio-chemical characteristics of the herbal formulations

\begin{tabular}{|c|c|c|c|c|c|}
\hline \multirow{2}{*}{ Formulation } & \multicolumn{3}{|c|}{ Ash value (\% w/w) } & \multicolumn{2}{|c|}{ Extractive value $(\% \mathrm{w} / \mathrm{w})$} \\
\hline & Total ash & Acid insoluble ash & Water soluble ash & Alcohol soluble extractive & Water soluble extractive \\
\hline $\begin{array}{c}\text { Gymnemic acid and } \\
\text { curcumin suspension (GCS) }\end{array}$ & 8.02 & 1.54 & 3.68 & 13.24 & 21.33 \\
\hline Hyponid tablet & 25.10 & 16.31 & 26.06 & 10.66 & 16.50 \\
\hline Madhumehari granules & 10.50 & 6.08 & 25.10 & 10.10 & 14.90 \\
\hline
\end{tabular}

Table 2: Co-TLC for identification of gymnemic acid and curcumin in the formulation

\begin{tabular}{|c|c|c|c|}
\hline \multirow{2}{*}{ Sample } & \multicolumn{3}{|c|}{ Solvent system chloroform: methanol: ethyl acetate $(5: 1: 1)$} \\
\hline & Rf value & No. of Spots & Inference \\
\hline Standard Gymnemic acid & 0.37 & 1 Spot & Well separated \\
\hline Isolated Gymnemic acid & 0.38 & 1 Spots & Well separated \\
\hline GCS & $0.38,0.44$ & 3 Spots & Well separated, light spot \\
\hline Hyponid & $0.38,0.46,0.55,60$ & 4 Spots & Separated, light spots \\
\hline Madhumehari & $0.39,0.42,0.55,61$ & 4 Spots & Well separated \\
\hline \multicolumn{4}{|c|}{ Solvent system chloroform: ethanol: acetic acid (94:4:1) } \\
\hline Standard curcumin & 0.47 & 1 Spot & Well separated \\
\hline Isolated curcumin & 0.46 & 3 Spots & Well separated \\
\hline GCS & $0.38,0.46,0.52$ & 3 Spots & Well separated \\
\hline Hyponid & $0.47,0.57,0.68$ & 3 Spots & Light spots \\
\hline Madhumehari & $0.40,0.46,0.58,0.64$ & 4 Spots & Light spots \\
\hline
\end{tabular}

Table 3: Effect of GCS, Hyponid and Madhumehari on relative weight of liver and kidney of alloxen induced diabetic in rat

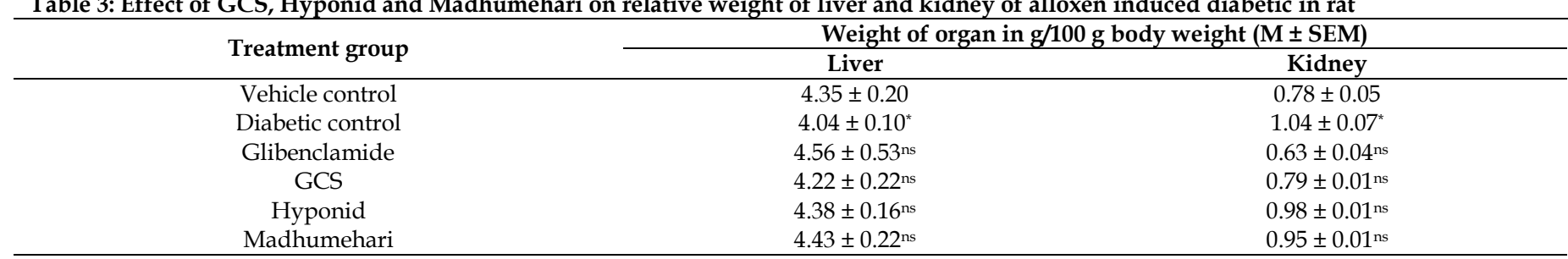

The values are expressed in $\mathrm{M} \pm$ SEM considering $\mathrm{n}=6$ per group. ${ }^{*} p<0.05$ and ns $=$ non significant when compared to vehicle control group. GCS = Gymnemic acid and curcumin suspension.

Table 4: Effect of GCS, Hyponid and Madhumehari on serum biochemical parameters of alloxan induced diabetic in rat

\begin{tabular}{|c|c|c|c|c|c|}
\hline \multirow{2}{*}{ Treatment group } & \multicolumn{5}{|c|}{ Lipid profile in $\mathrm{mg} / \mathrm{dl}(\mathrm{M} \pm \mathrm{SEM})$} \\
\hline & Total cholesterol & Triglyceride & HDL & LDL & VLDL \\
\hline Vehicle control & $122.33 \pm 6.71$ & $132.45 \pm 5.19$ & $54.70 \pm 3.11$ & $88.56 \pm 4.10$ & $26.49 \pm 2.33$ \\
\hline Diabetic control & $224.35 \pm 5.10^{* * *}$ & $168.23 \pm 5.50^{* * *}$ & $22.87 \pm 2.16^{* * *}$ & $167.70 \pm 5.40^{* * *}$ & $33.04 \pm 2.31^{*}$ \\
\hline Glibenclamide & $190.44 \pm 7.12^{*}$ & $154.95 \pm 3.84^{\mathrm{ns}}$ & $42.38 \pm 3.12^{* *}$ & $142.88 \pm 4.20^{\mathrm{ns}}$ & $30.98 \pm 1.16^{\mathrm{ns}}$ \\
\hline GCS & $185.39 \pm 8.11^{* *}$ & $153.44 \pm 5.92^{*}$ & $45.89 \pm 4.94^{* *}$ & $105.46 \pm 6.12^{* *}$ & $30.36 \pm 1.80^{*}$ \\
\hline Hyponid & $177.21 \pm 10.32^{* *}$ & $146.80 \pm 6.86^{*}$ & $50.54 \pm 3.38^{* * *}$ & $85.58 \pm 5.10^{* * *}$ & $29.77 \pm 2.36^{*}$ \\
\hline Madhumehari & $172.56 \pm 11.10^{* *}$ & $148.46 \pm 8.90^{*}$ & $50.82 \pm 4.98^{* * *}$ & $90.33 \pm 8.10^{* * *}$ & $29.35 \pm 1.20^{*}$ \\
\hline
\end{tabular}

The values are expressed in $\mathrm{M} \pm$ SEM considering $\mathrm{n}=6$ per group. ${ }^{* * *} p<0.001,{ }^{* *} p<0.01,{ }^{*} p<0.05$ and ns $=$ non-significant when compared to vehicle control group. The values in parenthesis signify the percentage change in blood glucose level of the respective group animal compared to 0 day value. $\mathrm{HDL}=$ high density lipoprotein, $\mathrm{LDL}=$ low density lipoprotein, VLDL = very low density lipoprotein and GCS = Gymnemic acid and curcumin suspension.

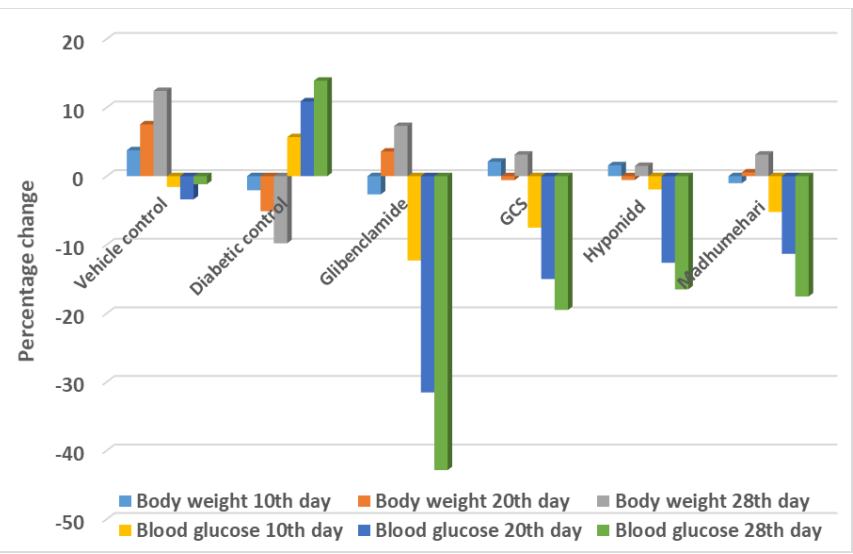

Fig. 3: Effect of GCS, Hyponid and Madhumehari on body weight and blood glucose level of alloxan induced diabetic in rat

\section{Effect on Biochemical Parameters}

The diabetic control group showed significantly $(\mathrm{P}<$ 0.05 - 0.001) higher serum TC, TG, LDL and VLDL compared to the vehicle control group. Compared to the vehicle control group, glibenclamide significantly reduced TC and increased HDL level but had a nonsignificant effect on TG, LDL, and VLDL. The GCS, Hyponid, and Madhumehari had efficiently $(p<0.05$ 0.001) decreased TC, TG, LDL and VLDL level along with an increase in HDL. Effect of Hyponid and Madhumehari was more pronounced on LDL and HDL level normalization in the diabetic rat group (Table 4).

\section{DISCUSSION}

A suspension was prepared with isolated curcumin and gymnemic acid and authenticated by TLC and HPTLC analysis. The GCS lowered blood glucose, TG, TC, and LDL level which was nearly equivalent as that of the marketed Polyherbal formulations Hyponid and Madhumehari against alloxan induced diabetes on rats following 28 days treatment. The potential 
hypoglycemic and hypolipidaemic effect of the GCS can be advantageous in treating diabetes associated hyperlipidaemic conditions considering its effects on lipid components. Standardization of traditional medicine used for therapeutic purposes has become extremely important looking into the current global herbal drug regulatory scenario. [18] The efficacy outcome of phytotherapy is based on the presence of multiple phytoconstituents in the polyherbal formulations composed of more than one plant having proven therapeutic values. [19] With this backdrop instead of using crude extract, the isolated bioactive phytocompounds has been selected to prepare a standardized antidiabetic formulation.

G. sylvestre leaf extract stimulates the pancreas to increase insulin release and fecal excretion of cholesterol in diabetic patient. [20] The primary bioactive principle in G. sylvestre is a triterpenoid saponin, gymnemic acid responsible for antihyperglycemic activity. Gymnemic acid promotes islet cells regeneration, increases insulin secretion, inhibit intestinal glucose absorption and increases utilization of glucose. [21] Turmeric has been used for thousands of years in Asian cookery and is a primary ingredient of curry powder. Alkaloid curcumin is responsible for the antidiabetic activity of C. longa extract. [22] Curcumin decreases blood sugar and cholesterol level apparently by blocking the enzymes that convert dietary carbohydrate to glucose. [23] The GCS was formulated with curcumin and gymnemic acid isolated from $C$. longa and G. sylvestre, and the antidiabetic effect was screened against alloxan on rat. The results indicated a gradual decrement in body weight gain in the diabetic group whereas treatment with GCS, Hyponid, and Madhumehari reversed the trend towards positive weight gain. All three formulations had normalized liver weight decrease and kidney weight increase as shown by the diabetic group. The relationship between body weight and relative organ weight (liver and kidney) with the associated risk of diabetes is successive and graded. [24] GCS, Hyponid, and Madhumehari have effectively normalized the body weight gain and also the relative weight of the liver and kidney of the diabetic animals.

The GCS significantly decreased blood glucose level in similar efficacy as off Hyponid and Madhumehari. Diabetic hyperglycemia is also accompanied with increase in serum cholesterol and triglyceride levels. ${ }^{[24]}$ Reduction in triglyceride through drug therapy has been found beneficial in preventing diabetic complications with improvement in lipid metabolism of diabetic patients. [25] Insulin deficiency results in impaired glucose utilization, causing hyperglycemia and mobilization of fatty acids from adipose tissue, in turn, increasing TG. [26] The GCS, Hyponid, and Madhumehari effectively reduced the elevated lipid parameters in diabetic rats. Insulin resistance in skeletal muscles promotes hepatic triglyceride synthesis which in turn generates large quantity of atherogenic triglyceride rich lipoprotein particles like VLDL. [27] GCS, Hyponid, and Madhumehari were equally effective in decreasing VLDL and increasing HDL of diabetic animals.

The isolated gymnemic acid and curcumin suspension were highly effective in lowering blood glucose, cholesterol, triglyceride, and LDL level. As GCS contains only two isolated compounds, it is expected to exert decreased metabolic bioburden with rare chances of side effect. In future, this current strategy can be applied for developing formulation containing two to four isolated phytocompounds followed by development of scientific evaluation data on quality, safety, and efficacy to get permission to proceed for clinical trial as per CDSCO, 2015 gazette notification for Phytopharmaceuticals. Development of HPLC method for simultaneous estimation and quantification of phytocompounds, and conduction of subacute and chronic exposure safety studies are required for complete profiling of the formulation required for regulatory approval.

The study showed that the standardized gymnemic acid and curcumin suspension possess potential antidiabetic activity, substantiating that the standardized bioactive phytocompound based suspension is equally efficacious compared to marketed Polyherbal formulations. This formulation also showed significant antihyperlipidemic activity by lowering serum cholesterol and triglyceride levels effectively. The phytocomponent formulation developed can be regarded as a promising natural and safe remedy for the prevention of diabetic complications. Further studies are required to establish long term safety profile and assessment of the effect on vital and metabolic organs. Thus following this study, it can be concluded that standardized bioactive phytocompound based formulations are equally efficacious to that of Polyherbal formulation.

\section{REFERENCES}

1. European Medicines Agency. Herbal medicinal products. https:/ / www.ema.europa.eu/en/human-regulatory/herbalmedicinal-products.

2. Sahoo N, Manchikanti P. Drug Regulation and Commercialization: An Indian Industry. Journal of Alternative and Complementary Medicine. 2013; 19(12): $957-$ 963. DOI: 10.1089/acm.2012.0275.

3. Anonymous. Diabetes is India's fastest growing disease: 72 million cases recorded in 2017, figure expected to nearly double by 2025.2018 . Available at: http:/ / www.firstpost.com/india/diabetes-is-indias-fastestgrowing-disease-72-million-cases-recorded-in-2017-figureexpected-to-nearly-double-by-2025-4435203.html.

4. Gupta R, Bajpai GK, Johri S, Saxena AM. An overview of Indian novel traditional medicinal plants with antidiabetic potentials. African Journal of Traditional, Complementary and Alternative Medicines. 2008; 5(1): 1-17.

5. Modak M, Dixit P, Londhe J, Ghaskadbi S, Devasagayam TPA. Indian herbs and herbal drugs used for the treatment of diabetes. Journal of Clinical Biochemistry and Nutrition. 2007; 40(3): 163-173. DOI: $10.3164 /$ jcbn.40.163. 
6. Subramani P, Gan ST, Dhanaraj SA. Polyherbal formulation: Concept of Ayurveda. Pharmacognosy Review. 2014; 8(16): 73-80. DOI: 10.4103/0973-7847.134229

7. Sharma AK, Gaurav SS, Balkrishna A. A rapid and simple scheme for the standardization of polyherbal drugs. International Journal of Green Pharmacy. 2009; 3(2): 134-140.

8. Kumar P, Rani S, Arunjyothi B, Chakrapani P, Rojarani A. Evaluation of antidiabetic activity of Gymnema sylvestre and Andrographis paniculata in streptozotocin induced diabetic Rats. International Journal of Pharmacognosy and Phytochemistry Research. 2017; 9(1): 22-25. DOI: 10.25258/ijpapr.v9i1.8035.

9. Mohammed A, Wudil AM, Alhassan AJ, Imam AA, Muhammad IU, Idi A. Hypoglycemic activity of Curcuma longa Linn root extracts on alloxan induced diabetic rats. Haya: Saudi Journal of Life Science. 2017; 2(2): 43-49. DOI 10.21276/haya.2017.2.2.4 43.

10. Chowdhary F, Rasool MH. Isolation and characterization of Gymnemic acid from indigenous Gymnema sylvestre. Journal of Applied Pharmacy. 2010; 3(2): 60-65. DOI: 10.21065/19204159.2.60.

11. Anonymous. Master document of Gymnema sylvestris. Natural Remedy Bangalore. 2010. Available at: https://www.naturalremedy.com/products/human.

12. Kokate CK, Purohit AP, Gokhale SB. A Textbook of Pharmacognosy. 39th Edn., Nirali prakashan, Pune. 2004; pp. 134-135. ISBN No: 9788185790152.

13. WHO. Quality control methods for medicinal plant material. Organization Mondiale De La Sante, Geneva. 1998. http://whqlibdoc.who.int/publications/1998/9241545100.p df.

14. The Ayurvedic Pharmacopoeia of India. Government of India Department of Ayurveda, Yoga \& Naturopathy, Unani, Siddha \& Homoeopathy (AYUSH). Part I, vol. 51th Edn., The Controller of Publications, New Delhi. 2006; pp. 143-144. ISBN No: 8190115138/9788190115131.

15. Malik JK, Manvi FV, Alagawadi KR, Noolvi M. Evaluation of anti-inflammatory activity of Gymnema sylvestre leaves extract in Rats. International Journal of Green Pharmacy. 2008; 2:

114-115. DOI: http://dx.doi.org/10.22377/ijgp.v2i2.42

16. Mall GK, Mishra PK, Prakash V. Antidiabetic and hypolipidemic activity of G. sylvestre in alloxan induced diabetes. Global Journal of Biotechnology and Biochemistry. 2009; 4(1): 37-42.

17. Friedewald WT, Levy RI, Fredrickson DS. Estimation of the concentration of low-density lipoprotein cholesterol in plasma, without use of the preparative ultracentrifuge. Clinical Chemistry. 1972; 18(6): 499-502.

18. Dwivedi C, Daspaul S. Antidiabetic herbal drugs and polyherbal formulation used for diabetes: A review. Journal of Phytopharmacology. 2013; 2(3): 44-51.

19. Miccoli R, Penno G, Del Parto S. Multidrug treatment of type 2 diabetes: A challenge for compliance. Diabetes Care. 2011; 34(Suppl 2): S231-S235. DOI: 10.2337/dc11-s235.

20. Kim HJ, Hong $\mathrm{SH}$, Chang $\mathrm{SH}$, Kim S, Lee AY, Jang $\mathrm{Y}$ Davaadamdin O, Yu KN, Kim JE, Cho MH. Effects of feeding a diet containing Gymnema sylvestre extract: Attenuating progression of obesity in C57BL/6J mice. Asian Pacific Journal Tropical Medical. 2016; 9(5): 437-444.

21. El Shafey AAM, El-Ezabi MM, Seliem MME, Ouda HHM, Ibrahim DS. Effect of Gymnema sylvestre R. Br. leaves extract on certain physiological parameters of diabetic rats. Journal of King Saud Univerity of Science. 2013; 25(2): 135-141.

22. Soetikno V, Watanabe K, Sari FR, Harima M, Thandavarayan RA, Veeraveedu PT, Arozal W, Sukumaran V, Lakshmanan AP, Arumugam S, Suzuki K. Curcumin attenuates diabetic nephropathy by inhibiting PKC- $\alpha$ and PKC- $\beta 1$ activity in streptozotocin-induced type I diabetic rats. Molecular Nutrition and Food Research. 2011; 55(11): 1655-1665. DOI: 10.1002/mnfr.201100080.

23. Seo KI, Choi MS, Jung UJ, Kim HJ, Yeo J, Jeon SM, Lee MK. Effect of curcumin supplementation on blood glucose, plasma insulin, and glucose homeostasis related enzyme activities in diabetic $\mathrm{db} / \mathrm{db}$ mice. Molecular Nutrition and Food Research. 2008; 52(9): 995-1004. DOI: 10.1002/mnfr.200700184.

24. Eleazu CO, Iroaganachi M, Okafor PN, Ijeh II, Eleazu KC. Ameliorative potentials of ginger ( $Z$. officinale Roscoe) on relative organ weights in streptozotocin induced diabetic rats. International Journal of Biomedical Science. 2013; 9(2): 82-90.

25. Vijayaraghavan K. Treatment of dyslipidemia in patients with type 2 diabetes. Lipids Health Disease. 2010; 9: 144 DOI: 10.1186/1476-511X-9-144

26. Ye J. Mechanisms of insulin resistance in obesity. Frontiers in Medicine. 2013; 7(1): 14-24. DOI: 10.1007/s11684-013-0262-6

27. Peterson KF, Dufour S, Savage DB, Bliz S, Soloman G, Yonemitsu S, Cline GW, Befroy D, Zemany L, Khan BB, Papademetris X, Rothman DL, Shulman GI. The role of skeletal muscles insulin resistance in the pathogenisis of the metabolic syndrome. Proceedings of the National Academy of Sciences of the United States of America. 2007; 104: 1258712594. DOI: $10.1073 /$ pnas.0705408104. 